\title{
Equilibrium existence under generalized convexity
}

\section{Monica Patriche}

\begin{abstract}
We introduce, in the first part, the notion of weakly convex pair of correspondences, we give its economic interpretation, we state a fixed point and a selection theorem. Then, by using a tehnique based on a continuous selection, we prove existence theorems of quilibrium for an abstract economy. In the second part, we define the weakly biconvex correspondences, we prove a selection theorem and we also demonstrate the existence of equilibrium for a generalized quasi-game (2003 Kim's model). In the last part of the paper, we give other applications in the game theory, finding equilibrium for abstract economies having correspondences with weakly convex graph. We show that the equilibrium exists without continuity assumptions.
\end{abstract}

\section{Introduction}

An open problem in the equilibrium theory is to prove the existence of fixed points for correspondences under nonconvexity (in the usual sense) assumptions. Some results on this subject were obtained by C. D. Horvath [7], G. Tian [13], X. Ding, He Yiran [3] or K. Wlodarczyk and D. Klim [15], [16]. The aim of this paper is to prove a fixed point and a selection theorem under generalized covexity conditions and to give an application in the game theory. The importance of these results also consists of the fact that the existence of

Key Words: weakly convex pairs of correspondence, weakly biconvex correspondences, weakly convex graph, fixed point theorem, continuous selection, abstract economy, equilibrium.

2010 Mathematics Subject Classification: 91B52, 91B50, 91A80.

Received: April, 2011.

Revised: April, 2011.

Accepted: February, 2012. 
fixed points and of the equilibrium takes place without continuity properties of the involved correspondences.

Within the last years, many authors generalized the classical models of abstract economy due to A. Borglin and H. Keiding [2], W. Shafer and H. Sonnenschein [12] or N. C. Yannelis and N. D. Prahbakar [18]. Also, W.K. Kim [8] obtained a generalization of the quasi fixed-point theorem due to I. Lefebvre [9] and, as an application, he proved an existence theorem of equilibrium for a generalized quasi-game with infinite number of agents. W.K.Kim's result concerns generalized quasi-games where the strategy sets are metrizable subsets in linear topological convex spaces.

In the first part of this paper we introduce the notion of weakly convex pair of correspondences, we give its economic interpretation, we state a fixed point and a selection theorem. Then, by using a tehnique based on a continuous selection, we prove existence theorems of quilibrium for an abstract economy. In the second part, we define the weakly biconvex correspondences, we prove a selection theorem and we also demonstrate the existence of equilibrium for a generalized quasi-game (2003 Kim's model). In the last part of the paper we give other applications in the game theory, finding equilibrium for abstract economies having correspondences with weakly convex graph. We show that the equilibrium exists without continuity assumptions.

Bi-convexity was studied by R. Aumann, S. Hart in [1] or J. Gorski, F. Pfeuffer, K. Klamroth in [5]. We continue our work on studying the existence conditions of equilibrium of quasi-games [10] or the existence of fixed points for correspondences [11].

The paper is organized in the following way: Section 2 contains preliminaries and notation. The weakly convex pairs of correspondences are studied in Section 3. Biconvexity of the correspondences, W. K. Kim's model of quasigame and the quasi-equilibrium existence results are presented in Section 4. The equilibrium theorems for correspondences with the weakly convex graph selection property are stated in Section 5 .

\section{Preliminaries and notation}

Let $A$ be a subset of a topological space $X .2^{A}$ denotes the family of all subsets of $A$. cl $A$ denotes the closure of $A$ in $X$. If $A$ is a subset of a vector space, co $A$ denotes the convex hull of $A$. If $F, T: A \rightarrow 2^{X}$ are correspondences, then $\operatorname{co} T, \operatorname{cl} T, T \cap F: A \rightarrow 2^{X}$ are correspondences defined by $(\operatorname{co} T)(x)=\operatorname{co} T(x)$, $(\operatorname{cl} T)(x)=\operatorname{cl} T(x)$ and $(T \cap F)(x)=T(x) \cap F(x)$ for each $x \in A$, respectively. The graph of $T: X \rightarrow 2^{Y}$ is the set $\operatorname{Gr}(T)=\{(x, y) \in X \times Y \mid y \in T(x)\}$. 
The correspondence $\bar{T}$ is defined by $\bar{T}(x)=\left\{y \in Y:(x, y) \in \mathrm{cl}_{X \times Y} \operatorname{Gr} T\right\}$ (the set $\operatorname{cl}_{X \times Y} \operatorname{Gr}(T)$ is called the adherence of the graph of $T$ ). It is easy to see that $\operatorname{cl} T(x) \subset \bar{T}(x)$ for each $x \in X$.

Lemma 1. [19] Let $X$ be a topological space, $Y$ be a non-empty subset of a topological vector space $E, \beta$ be a base of the neighborhoods of 0 in $E$ and $A: X \rightarrow 2^{Y}$. For each $V \in \beta$, let $A_{V}: X \rightarrow 2^{Y}$ be defined by $A_{V}(x)=(A(x)+$ $V) \cap Y$ for each $x \in X$. If $\widehat{x} \in X$ and $\widehat{y} \in Y$ are such that $\widehat{y} \in \cap_{V \in \beta} \overline{A_{V}}(\widehat{x})$, then $\widehat{y} \in \bar{A}(\widehat{x})$.

Definition 1. Let $X, Y$ be topological spaces and $T: X \rightarrow 2^{Y}$ be a correspondence

1. $T$ is said to be upper semicontinuous if for each $x \in X$ and each open set $V$ in $Y$ with $T(x) \subset V$, there exists an open neighborhood $U$ of $x$ in $X$ such that $T(y) \subset V$ for each $y \in U$.

2. $T$ is said to be lower semicontinuous if for each $\mathrm{x} \in X$ and each open set $V$ in $Y$ with $T(x) \cap V \neq \emptyset$, there exists an open neighborhood $U$ of $x$ in $X$ such that $T(y) \cap V \neq \emptyset$ for each $y \in U$.

Lemma 2. [14] Let $X$ be a topological space, $Y$ be a topological linear space, and let $A: X \rightarrow 2^{Y}$ be an upper semicontinuous correspondence with compact values. Assume that the sets $C \subset Y$ and $K \subset Y$ are closed and respectively compact. Then $T: X \rightarrow 2^{Y}$ defined by $T(x)=(A(x)+C) \cap K$ for all $x \in X$ is upper semicontinuous.

To prove our theorems, we need Wu's theorem:

Theorem 3. [17] Let I be an index set. For each $i \in I$, let $X_{i}$ be a nonempty convex subset of a Hausdorff locally convex topological vector space $E_{i}, D_{i}$ a non-empty compact metrizable subset of $X_{i}$ and $S_{i}, T_{i}: X:=\prod_{i \in I} X_{i} \rightarrow 2^{D_{i}}$ two correspondences with the following conditions:

(i) for each $x \in X, \overline{\mathrm{co}} S_{i}(x) \subset T_{i}(x)$ and $S_{i}(x) \neq \emptyset$,

(ii) $S_{i}$ is lower semicontinuous.

Then, there exists a point $\bar{x}=\prod_{i \in I} \bar{x}_{i} \in D=\prod_{i \in I} D_{i}$ such that $\bar{x}_{i} \in T_{i}(\bar{x})$ for each $i \in I$. 


\section{Weakly convex pairs of correspondences}

Notation. Let $\Delta_{n-1}=\left\{\left(\lambda_{1}, \lambda_{2}, \ldots, \lambda_{n}\right) \in \mathbb{R}^{n}: \sum_{i=1}^{n} \lambda_{i}=1\right.$ and $\lambda_{i} \geqslant 0, i=$ $1,2, \ldots$,

$n$ \} be the standard (n-1)-dimensional simplex in $\mathbb{R}^{n}$.

We introduce the following notion.

Definition 2. Let $X$ be a convex set in a topologiacl vector space $E, Y$ be a nonempty subset of a topological vector $F$ and $S, T: X \rightarrow 2^{Y}$ two correspondences. $(S, T)$ is called weakly convex pair of correspondences if, for each finite set $\left\{x_{1}, x_{2}, \ldots, x_{n}\right\} \subset X$, there exists $y_{i} \in S\left(x_{i}\right),(i=1,2, \ldots, n)$ such that for every $\lambda_{1}, \lambda_{2}, \ldots, \lambda_{n} \in \Delta_{n-1}$, then $y=\sum_{i=1}^{n} \lambda_{i} y_{i} \in T\left(\sum_{i=1}^{n} \lambda_{i} x_{i}\right)$.

\subsection{A fixed point theorem}

We state the following fixed point theorem:

Theorem 4. Let $Y$ be a non-empty subset of a topological vector space $E$ and $K$ be a $(n-1)$ - dimensional simplex in $E$. Let $(S, T): K \rightarrow 2^{Y}$ be a weakly convex pair of correspondences and $s: Y \rightarrow K$ be a continuous function. Then, there exists $x^{*} \in K$ such that $x^{*} \in s \circ T\left(x^{*}\right)$.

Proof. Let $a_{1}, a_{2}, \ldots, a_{n}$ be the vertices of $K$. Since $(S, T)$ is weakly convex pair of correspondences, there exist $b_{i} \in S\left(a_{i}\right)$, such that for every $\left(\lambda_{1}, \lambda_{2}, \ldots, \lambda_{n}\right) \in$ $\Delta_{n-1}$, then $y=\sum_{i=1}^{n} \lambda_{i} b_{i} \in T\left(\sum_{i=1}^{n} \lambda_{i} a_{i}\right)$.

Since $K$ is a $(n-1)$-dimensional simplex with the vertices $a_{1}, \ldots, a_{n}$, there exists unique continuous functions $\lambda_{i}: K \rightarrow \mathbb{R}, i=1,2, \ldots, n$ such that for each $x \in K$, we have $\left(\lambda_{1}(x), \lambda_{2}(x), \ldots, \lambda_{n}(x)\right) \in \Delta_{n-1}$ and $x=\sum_{i=1}^{n} \lambda_{i}(x) a_{i}$.

Let's define $f: K \rightarrow 2^{Y}$ by

$f\left(a_{i}\right)=b_{i}(i=1, \ldots, n)$ and

$f\left(\sum_{i=1}^{n} \lambda_{i} a_{i}\right)=\sum_{i=1}^{n} \lambda_{i} b_{i} \in T\left(\sum_{i=1}^{n} \lambda_{i} a_{i}\right)$.

We show that $f$ is continuous.

Let $\left(x_{m}\right)_{m \in N}$ be a sequence which converges to $x_{0} \in K$, where $x_{m}=$ $\sum_{i=1}^{n} \lambda_{i}\left(x_{m}\right) a_{i}$ and $x_{0}=\sum_{i=1}^{n} \lambda_{i}\left(x_{0}\right) a_{i}$. By the continuity of $\lambda_{i}$, it follows that for each $i=1,2, \ldots, n, \lambda_{i}\left(x_{m}\right) \rightarrow \lambda_{i}\left(x_{0}\right)$ as $m \rightarrow \infty$. Hence $f\left(x_{m}\right) \rightarrow f\left(x_{0}\right)$ as $m \rightarrow \infty$, i.e. $f$ is continuous. 
Since $s: Y \rightarrow K$ is continuous, we obtain that $s \circ f: K \rightarrow K$ is continuous. According to Brouwer's fixed point theorem, there exists a point $x^{*} \in K$ such that $x^{*}=s \circ f\left(x^{*}\right)$ and then, $x^{*} \in s \circ T\left(x^{*}\right)$.

Theorem 5. (selection theorem). Let $Y$ be a non-empty subset of a topological vector space $E$ and $K$ be $a(n-1)$-dimensional simplex in a topological vector space $F$. Let $(S, T): K \rightarrow 2^{Y}$ be a weakly convex pair of correspondences. Then, $T$ has a continuous selection on $K$.

\subsection{Economic interpretation}

We consider an abstract economy with $I$ - the set of agents. Each agent can choose a strategy from a set $X_{i}$ and has a preferrence correspondence $P_{i}: X=$ $\prod_{i \in I} X_{i} \rightarrow X_{i}$ and a constraint correspondence $A_{i}: X=\prod_{i \in I} X_{i} \rightarrow 2^{X_{i}}$. The traditional approach considers that the preferrence of agent $i$ is characterized by a binary relation $\succeq_{i}$ on the set $X_{i}$. A real valued function $u_{i}$ that satisfies $x \succeq_{i} y \Leftrightarrow u_{i}(x) \geq u_{i}(y)$ is called an utility function of the preferrence $\succeq_{i}$. The relation between the utility function $u_{i}$ and the preferrence correspondence for each agent $i$ is:

$P_{i}(x)=\left\{y_{i} \in A_{i}(x): u_{i}\left(x, y_{i}\right)>u_{i}\left(x, x_{i}\right)\right\}$, where, in this case, $u_{i}: X \times$ $X_{i} \rightarrow X_{i}$.

The aim of the equilibrium theory is to maximize each agent's utility on a convex strategy set. For that, the notion of convexity of the preferrence is very important:

Definition 3. The preferrence $\succeq$ is called convex if $x \succeq y$ implies $\lambda x+(1-$ $\lambda) y \succeq y$ for $\lambda \in[0,1]$.

The intuitive interpretation is that, given two strategies $x$ and $y$, the composed strategy $\lambda x+(1-\lambda) y \succeq y$ with $\lambda \in[0,1]$ is more valuable if $x$ is already preferrable to $y$. For an abstract economy, if we have $y_{i} \in A_{i}(x)$ and $u_{i}\left(x, y_{i}\right)>u_{i}\left(x, x_{i}\right)$, if the preferrence $\succeq_{i}$ (and then the utility function $u_{i}$ ) is convex, we obtain that

if $y_{i} \in A_{i}(x)$ then $u_{i}\left(x, \lambda y_{i}+(1-\lambda) x_{i}\right)>u_{i}\left(x, x_{i}\right)$

or, equivalently,

if $y_{i} \in P_{i}(x)$ then $\lambda y_{i}+(1-\lambda) x_{i} \in P_{i}(x)$ if we have that $\lambda y_{i}+(1-\lambda) x_{i} \in$ $A_{i}(x)$.

For the case that, for the index $i,\left(A_{i}, P_{i}\right)$ is a weakly convex pair of correspondences, the interpretation is the following: for every $x^{1}, x^{2}, \ldots, x^{n} \in$ $X$, there exist $y_{i}^{1} \in A_{i}\left(x^{1}\right), y_{i}^{2} \in A_{i}\left(x^{2}\right), \ldots, y_{i}^{n} \in A_{i}\left(x^{n}\right)$, such that, for each $\lambda \in$ 
$\Delta_{n-1}$, there exists $y_{i}=\sum_{k=1}^{n} \lambda_{k} y_{i}^{k}$ with the property that $y_{i} \in P_{i}\left(\sum_{k=1}^{n} \lambda_{k} x^{k}\right)$ (i.e., if there exists the utility function $u_{i}: y_{i} \in A_{i}\left(\sum_{k=1}^{n} \lambda_{k} x^{k}\right)$ and $u_{i}\left(\sum_{k=1}^{n} \lambda_{k} x^{k}, y_{i}\right)>$ $u_{i}\left(\sum_{k=1}^{n} \lambda_{k} x^{k},\left(\sum_{k=1}^{n} \lambda_{k} x^{k}\right)_{i}\right)$

We introduce the notion of weakly convex preferrence.

Definition 4. The preferrence $\succeq$ is called weakly convex if for each $y \in X$, there exists $x \in X$ such that for each $\lambda \in[0,1]$ we have that $\lambda x+(1-\lambda) y \succeq y$.

\subsection{Applications in the equilibrium theory}

First, we present the model of an abstract economy and the definition of an equilibrium.

Let $I$ be a non-empty set (the set of agents). For each $i \in I$, let $X_{i}$ be a non-empty topological vector space representing the set of actions and let's define $X:=\prod_{i \in I} X_{i}$; let $A_{i}, B_{i}: X \rightarrow 2^{X_{i}}$ be the constraint correspondences and $P_{i}$ the preference correspondence.

Definition 5. The family $\Gamma=\left(X_{i}, A_{i}, P_{i}, B_{i}\right)_{i \in I}$ is said to be an abstract economy.

Definition 6. An equilibrium for $\Gamma$ is defined as a point $x^{*} \in X$ such that for each $i \in I, x_{i}^{*} \in \bar{B}_{i}\left(x^{*}\right)$ and $A_{i}\left(x^{*},\right) \cap P_{i}\left(x^{*}\right)=\emptyset$.

Remark 1. When for each $i \in I, A_{i}(x)=B_{i}(x)$ for all $x \in X$, this abstract economy model coincides with the classical one introduced by Borglin and Keiding in [2]. If in addition, $\bar{B}_{i}\left(x^{*}\right)=c_{X_{i}} B_{i}\left(x^{*}\right)$ for each $x \in X$, which is the case if $B_{i}$ has a closed graph in $X \times X_{i}$, the definition of an equilibrium coincides with that one used by Yannelis and Prabhakar [18].

For the following theorems, we will use the selection theorem and a tehnique based on a continuous selection. We show the existence of equilibrium for an abstract economy without assuming the continuity of the constraint and of the preference correspondences $A_{i}$ and $P_{i}$.

First, we prove a new equilibrium existence theorem for a noncompact abstract economy with constraint and preference correspondences $A_{i}$ and $P_{i}$, which have the property that their intersection $A_{i} \cap P_{i}$ contains a selector $S_{i}$ on the domain $W_{i}$ of $A_{i} \cap P_{i},\left(A_{i}, S_{i}\right)$ is a weakly convex pair of correspondences and $W_{i}$ must be a simplex. To find the equilibrium point, we use Wu's fixed point theorem [17]. 
Theorem 6. Let $\Gamma=\left(X_{i}, A_{i}, P_{i}, B_{i}\right)_{i \in I}$ be an abstract economy, where $I$ is a (possibly uncountable) set of agents such that for each $i \in I$ :

(1) $X_{i}$ is a non-empty convex set in a locally convex space $E_{i}$ and there exists a compact subset $D_{i}$ of $X_{i}$ containing all the values of the correspondences $A_{i}, P_{i}$ and $B_{i}$ such that $D=\prod_{i \in I} D_{i}$ is metrizable;

(2) $\mathrm{cl} B_{i}$ is lower semicontinuous, has non-empty convex values and for each $x \in X, A_{i}(x) \subset B_{i}(x)$;

(3) $W_{i}=\left\{x \in X /\left(A_{i} \cap P_{i}\right)(x) \neq \emptyset\right\}$ is a $\left(n_{i}-1\right)$-dimensional simplex in $X$ such that $W_{i} \subset \mathrm{co} D$;

(4) there exists a correspondence $S_{i}: W_{i} \rightarrow 2^{D_{i}}$ such that $S_{i}(x) \subset\left(A_{i} \cap P_{i}\right)(x)$ for each $x \in W_{i}$ and $\left(A_{i}, S_{i}\right)$ is a weakly convex pair of correspondences;

(5) for each $x \in W_{i}, x_{i} \notin\left(A_{i} \cap P_{i}\right)(x)$.

Then there exists an equilibrium point $x^{*} \in D$ for $\Gamma$, i.e., for each $i \in I$, $x_{i}^{*} \in \operatorname{cl} B_{i}\left(x^{*}\right)$ and $A_{i}\left(x^{*}\right) \cap P_{i}\left(x^{*}\right)=\emptyset$.

Proof. Let be $i \in I$. From the assumption (4) and the selection theorem 3, it follows that there exists a continuous function $f_{i}: W_{i} \rightarrow D_{i}$ such that for each $x \in W_{i}, f_{i}(x) \in S_{i}(x) \subset A_{i}(x) \cap P_{i}(x) \subset B_{i}(x)$.

Let's define the correspondence $T_{i}: X \rightarrow 2^{D_{i}}$, by $T_{i}(x):=\left\{\begin{array}{l}\left\{f_{i}(x)\right\}, \text { if } x \in W_{i}, \\ \operatorname{cl} B_{i}(x), \text { if } x \notin W_{i}\end{array} ;\right.$

$T_{i}$ is lower semicontinuous on $X$.

Let $V$ be an closed subset of $X_{i}$, then

$U:=\left\{x \in X \mid T_{i}(x) \subset V\right\} \quad=\left\{x \in W_{i} \mid T_{i}(x) \subset V\right\} \cup\left\{x \in X \backslash W_{i} \mid\right.$ $\left.T_{i}(x) \subset V\right\}$

$$
\begin{aligned}
& =\left\{x \in W_{i} \mid f_{i}(x) \in V\right\} \cup\left\{x \in X \mid \operatorname{cl} B_{i}(x) \subset V\right\} \\
& =\left(f_{i}^{-1}(V) \cap W_{i}\right) \cup\left\{x \in X \mid \operatorname{cl} B_{i}(x) \subset V\right\} .
\end{aligned}
$$

$U$ is a closed set, because $W_{i}$ is closed, $f_{i}$ is a continuous map on $\operatorname{int}_{X} K_{i}$ and the set $\left\{x \in X \mid \operatorname{cl} B_{i}(x) \subset V\right\}$ is closed since $\operatorname{cl} B_{i}$ is l.s.c. Let $D=\prod_{i \in I} D_{i}$. Then, according to Tychonoff's Theorem, $D$ is compact in the convex set $X$.

By Wu's fixed-point theorem in [17], applied for the correspondences $S_{i}=$ $T_{i}$ and $T_{i}: X \rightarrow 2^{D_{i}}$, there exists $x^{*} \in D$ such that for each $i \in I, x_{i}^{*} \in T_{i}\left(x^{*}\right)$. If $x^{*} \in W_{i}$ for some $i \in I$, then $x_{i}^{*}=f_{i}\left(x^{*}\right)$, which is a contradiction.

Therefore, $x^{*} \notin W_{i}$, and hence $\left(A_{i} \cap P_{i}\right)\left(x^{*}\right)=\emptyset$. Also, for each $i \in I$, we have $x_{i}^{*} \in T_{i}\left(x^{*}\right)$, and then $x_{i}^{*} \in \operatorname{cl} B_{i}\left(x^{*}\right)$.

For Theorem 5, we use an approximation method, in the meaning that we obtain, for each $i \in I$, a continuous selection $f_{i}^{V_{i}}$ of $\left(A_{i}+V_{i}\right) \cap P_{i}$, where $V_{i}$ is a convex neighborhood of 0 in $X_{i}$. For every $V=\prod_{i \in I} V_{i}$, we obtain an equilibrium point for the associated approximate abstract economy $\Gamma_{V}=$ $\left(X_{i}, A_{i}, P_{i}, B_{V_{i}}\right)_{i \in I}$, i.e., a point $x^{*} \in X$ such that $A_{i}\left(x^{*}\right) \cap P_{i}\left(x^{*}\right)=\emptyset$ and $x_{i}^{*} \in B_{V_{i}}\left(x^{*}\right)$, where the correspondence $B_{V_{i}}: X \rightarrow 2^{X_{i}}$ is defined by 
$B_{V_{i}}(x)=\operatorname{cl}\left(B_{i}(x)+V_{i}\right) \cap X_{i}$ for each $x \in X$ and for each $i \in I$. Finally, we use Lemma 1 to get an equilibrium point for $\Gamma$ in $X$. The compactness assumption for $X_{i}$ is essential in the proof.

Theorem 7. Let $\Gamma=\left(X_{i}, A_{i}, P_{i}, B_{i}\right)_{i \in I}$ be an abstract economy, where $I$ is a (possibly uncountable) set of agents such that for each $i \in I$ :

(1) $X_{i}$ is a non-empty compact convex set in a locally convex space $E_{i}$;

(2) $\mathrm{cl}_{i}$ is upper semicontinuous, has non-empty convex values and for each $x \in X, A_{i}(x) \subset B_{i}(x)$;

(3) the set $W_{i}:=\left\{x \in X /\left(A_{i} \cap P_{i}\right)(x) \neq \emptyset\right\}$ is non-empty, open and $K_{i}=\mathrm{cl}_{i}$ is a $\left(n_{i}-1\right)$-dimensional simplex in $X$;

(4) For each convex neighbourhood $V$ of 0 in $X_{i},\left(A_{i},\left(A_{i}+V\right) \cap P_{i}\right)$ is a weakly convex pair of correspondences, where $\left(A_{i}+V\right) \cap P_{i}: K_{i} \rightarrow 2^{X_{i}}$;

(5) for each $x \in K_{i}, x_{i} \notin P_{i}(x)$.

Then there exists an equilibrium point $x^{*} \in X$ for $\Gamma$, i.e., for each $i \in I$, $x_{i}^{*} \in \bar{B}_{i}\left(x^{*}\right)$ and $A_{i}\left(x^{*}\right) \cap P_{i}\left(x^{*}\right)=\emptyset$.

Proof. For each $i \in I$, let $\beta_{i}$ denote the family of all open convex neighborhoods of zero in $E_{i}$. Let $V=\left(V_{i}\right)_{i \in I} \in \prod_{i \in I} \beta_{i}$. Since $\left(A_{i},\left(A_{i}+V\right) \cap P_{i}\right)$ is a weakly convex pair of correspondences on $K_{i}$, then, from the selection theorem 3 , there exists a continuous function $f_{i}^{V_{i}}: K_{i} \rightarrow X_{i}$ such that for each $x \in K_{i}$,

$f_{i}^{V_{i}}(x) \in\left(A_{i}(x)+V_{i}\right) \cap P_{i}(x) \subset\left(A_{i}(x)+V_{i}\right) \cap X_{i}$.

It follows that $f_{i}^{V_{i}}(x) \in \operatorname{cl}\left(B_{i}(x)+V_{i}\right)$ for $x \in K_{i}$. Since $X_{i}$ is compact, we have that $\operatorname{cl}\left(B_{i}(x)\right)$ is compact for every $x \in X$ and $\operatorname{cl}\left(B_{i}(x)+V_{i}\right)=\operatorname{cl}\left(B_{i}(x)\right)+\operatorname{cl} V_{i}$ for every $V_{i} \subset E_{i}$.

Let's define the correspondence $T_{i}^{V_{i}}: X \rightarrow 2^{X_{i}}$, by

$$
T_{i}^{V_{i}}(x):=\left\{\begin{array}{c}
\left\{f_{i}^{V_{i}}(x)\right\}, \quad \text { if } x \in \operatorname{int}_{X} K=W_{i}, \\
\operatorname{cl}\left(B_{i}(x)+V_{i}\right) \cap X_{i}, \text { if } x \in X \backslash \operatorname{int}_{X} K_{i}
\end{array}\right.
$$

The correspondence $B_{V_{i}}: X \rightarrow 2^{X_{i}}$, defined by $B_{V_{i}}(x):=\operatorname{cl}\left(B_{i}(x)+V_{i}\right) \cap X_{i}$ is u.s.c. by Theorem 1.1 in [14]. Then following the same line as in Theorem 4 , we can prove that $T_{i}^{V_{i}}$ is upper semicontinuous on $X$ and has closed convex values.

Let's define $T^{V}: X \rightarrow 2^{X}$ by $T^{V}(x):=\prod_{i \in I} T_{i}^{V_{i}}(x)$ for each $x \in X$.

$T^{V}$ is an upper semicontinuous correspondence and also has non-empty convex closed values.

Since $X$ is a compact convex set, according to Fan's fixed-point Theorem [4], there exists $x_{V}^{*} \in X$ such that $x_{V}^{*} \in T^{V}\left(x_{V}^{*}\right)$, i.e., for each $i \in I,\left(x_{V}^{*}\right)_{i} \in$ $T_{i}^{V_{i}}\left(x_{V}^{*}\right)$.

We state that $x_{V}^{*} \in X \backslash \bigcup_{i \in I} \operatorname{int}_{X} K_{i}$. 
If $x_{V}^{*} \in \operatorname{int}_{X} K_{i},\left(x_{V}^{*}\right)_{i} \in T_{i}^{V_{i}}\left(x_{V}^{*}\right)=f_{i}\left(x_{V}^{*}\right) \in\left(\left(A_{i}\left(x_{V}^{*}\right)+V_{i}\right) \cap P_{i}\right)\left(x_{V}^{*}\right) \subset$ $P_{i}\left(x_{V}^{*}\right)$, which contradicts assumption (5). where

Hence $\left(x_{V}^{*}\right)_{i} \in \operatorname{cl}\left(B_{i}\left(x_{V}^{*}\right)+V_{i}\right) \cap X_{i}$ and $\left(A_{i} \cap P_{i}\right)\left(x_{V}^{*}\right)=\emptyset$, i.e. $x_{V}^{*} \in Q_{V}$

$Q_{V}=\cap_{i \in I}\left\{x \in X: x_{i} \in \operatorname{cl}\left(B_{i}(x)+V_{i}\right) \cap X_{i}\right.$ and $\left.\left(A_{i} \cap P_{i}\right)(x)=\emptyset\right\}$.

Since $W_{i}$ is open, $Q_{V}$ is the intersection of non-empty closed sets, then it is non-empty, closed in $X$.

We prove that the family $\left\{Q_{V}: V \in \prod_{i \in I} \beta_{i}\right\}$ has the finite intersection property.

Let $\left\{V^{(1)}, V^{(2)}, \ldots V^{(n)}\right\}$ be any finite set of $\prod_{i \in I} \beta_{i}$ and let $V^{(k)}=\left(V_{i}^{(k)}\right)_{i \in I}$, $k=1, \ldots n$. For each $i \in I$, let $V_{i}=\bigcap_{k=1}^{n} V_{i}^{(k)}$, then $V_{i} \in \beta_{i}$; thus $V=\left(V_{i}\right)_{i \in I} \in$ $\prod_{i \in I} B_{i}$. Clearly $Q_{V} \subset \bigcap_{k=1}^{n} Q_{V^{(k)}}$ so that $\bigcap_{k=1}^{n} Q_{V^{(k)}} \neq \emptyset$.

Proof. Since $\mathrm{X}$ is compact and the family $\left\{Q_{V}: V \in \prod_{i \in I} \beta_{i}\right\}$ has the finite intersection property, we have that $\cap\left\{Q_{V}: V \in \prod_{i \in I} \beta_{i}\right\} \neq \emptyset$. Let's take any $x^{*} \in \cap\left\{Q_{V}: V \in \prod_{i \in I} \beta_{i}\right\}$, then for each $i \in I$ and each $V_{i} \in B_{i}, x_{i}^{*} \in \operatorname{cl}\left(B_{i}\left(x^{*}\right)+\right.$ $\left.V_{i}\right) \cap X_{i}$ and $\left(A_{i} \cap P_{i}\right)\left(x^{*}\right)=\emptyset$; but then $x^{*} \in \operatorname{cl}\left(B_{i}\left(x^{*}\right)\right)$ according to Lemma 1 and $\left(A_{i} \cap P_{i}\right)\left(x^{*}\right)=\emptyset$ for each $i \in I$ so that $x^{*}$ is an equilibrium point of $\Gamma$ in $\mathrm{X}$.

In the theorem above, the correspondences $A_{i} \cap P_{i}$ don't verify continuity assumptions and do not have convex or compact values. The importance of our results also consists in the fact that the existence of fixed points and of the equilibrium takes place without continuity properties of the correspondences involved.

\section{Biconvexity of the correspondences and applications in the game theory}

\subsection{Preliminaries}

Let $X \subset E_{1}$ and $Y \subset E_{2}$ be two nonempty, convex sets, $E_{1}, E_{2}$ are topological vector space and let $B \subset X \times Y$. The $y-$ and $x$ - sections of $B$ are defined as follows:

$$
\begin{aligned}
& B_{x}:=\{y \in Y:(x, y) \in B\} \\
& B_{y}:=\{x \in X:(x, y) \in B\}
\end{aligned}
$$


Definition 7. The set $B \subset X \times Y$ is called a biconvex set on $X \times Y$ if $B_{x}$ is convex for every $x \in X$ and $B_{y}$ is convex for every $y \in Y$.

Definition 8. Let $\left(x_{i}, y_{i}\right) \in X \times Y$ for $i=1,2, \ldots n$. A convex combination $(x, y)=\sum_{i=1}^{n} \lambda_{i}\left(x_{i}, y_{i}\right)$, (with $\left.\sum_{i=1}^{n} \lambda_{i}=1, \lambda_{i} \geq 0 i=1,2, \ldots, n\right)$ is called biconvex combination if $x_{1}=x_{2}=\ldots=x_{n}=x$ or $y_{1}=y_{2}=\ldots=y_{n}=y$.

The following characterization for biconvex sets was formulated by $\mathrm{Au}-$ mann and Hart:

Theorem 8. [1] $A$ set $B \subseteq X \times Y$ is biconvex if and only if $B$ contains all biconvex combinations of its elements.

As in the convex case, it is possible to define the biconvex hull of a given set $A \subseteq X \times Y$.

Definition 9. Let $A \subseteq X \times Y$ be a given set. The set $H:=\left\{\bigcap A_{I}: A \subseteq A_{I}\right.$,

$A_{I}$ is biconvex $\}$ is called the biconvex hull of $A$ and is denoted biconv $(A)$.

Aumann and Hart stated the following properties of the set $H$ :

Theorem 9. [1] The above defined set is biconvex. Furthermore, $H$ is the smallest biconvex set (in the sense of set inclusion), which contains $A$.

As biconvex combinations are, by definition, a special case of convex combinations and the convex hull $\operatorname{conv}(A)$ of a given set $A$ consists of all convex combinations of the elements of $A$, we have:

Lemma 10. Let $A \subseteq X \times Y$ be a given set. Then biconv $(A) \subseteq \operatorname{conv}(A)$.

Aumann and Hart proposed an inductively way to construct the biconvex hull of a given set $A$. They defined the sequence $\left\{A_{n}\right\}_{n \in N}$ as follows:

$A_{1}:=A$

$A_{n+1}:=\left\{(x ; y) \in A_{n}:(x, y)\right.$ is a biconvex combination of elements of $\left.A_{n}\right\}$.

Let $H^{\prime}:=\cup_{n \in N} A_{n}$ denote the limit of this sequence.

Proposition 11. [1] The above constructed set $H^{\prime}$ is biconvex and equals $H$, the biconvex hull of $A$.

We introduce the following definition. 
Definition 10. Let $B \subset X \times Y$ be a biconvex set, $Z$ a nonempty subset of a topological vector space $F$ and $T: B \rightarrow 2^{Z}$ a correspondence. $T$ is called weakly biconvex if for each finite set $\left\{\left(x_{1}, y_{1}\right),\left(x_{2}, y_{2}\right), \ldots,\left(x_{n}, y_{n}\right)\right\} \subset B$, there exists $z_{i} \in T\left(x_{i}, y_{i}\right),(i=1,2, \ldots, n)$ such that for every biconvex combination $(x, y)=\sum_{i=1}^{n} \lambda_{i}\left(x_{i}, y_{i}\right) \in B\left(\right.$ with $\left.\sum_{i=1}^{n} \lambda_{i}=1, \lambda_{i} \geq 0 i=1,2, \ldots, n\right)$, then $y=\sum_{i=1}^{n} \lambda_{i} z_{i} \in T\left(\sum_{i=1}^{n} \lambda_{i}\left(x_{i}, y_{i}\right)\right)$.

We formulate the following fixed point theorem for weakly biconvex correspondences.

Theorem 12. Let $Y$ be a non-empty subset of a topological vector space $F$ and $K \subset E_{1} \times E_{2}$, where $E_{1}, E_{2}$ are topological vector spaces. Suppose that $K$ is the biconvex hull of $\left\{\left(a_{1}, b_{1}\right),\left(a_{2}, b_{2}\right), \ldots,\left(a_{n}, b_{n}\right)\right\} \subset E_{1} \times E_{2}$. Let $T: K \rightarrow 2^{Y}$ be a weakly biconvex correspondence and $s: Y \rightarrow K$ be a continuous function. Then, there exists $x^{*} \in K$ such that $x^{*} \in s \circ T\left(x^{*}\right)$.

Proof. Since $T$ is weakly biconvex, there exist $c_{i} \in T\left(a_{i}, b_{i}\right),(i=1,2, \ldots, n)$, such that, for every $\left(\lambda_{1}, \lambda_{2}, \ldots, \lambda_{n}\right) \in \Delta_{n-1}$, there exists $z \in T\left(\sum_{i=1}^{n} \lambda_{i}\left(a_{i}, b_{i}\right)\right)$ with $z=\sum_{i=1}^{n} \lambda_{i} z_{i}$

Since $K$ is the biconvex hull of $\left(a_{1}, b_{1}\right), \ldots,\left(a_{n}, b_{n}\right)$, there exists unique continuous functions $\lambda_{i}: K \rightarrow \mathbb{R}, i=1,2, \ldots, n$ such that for each $(x, y) \in K$, we have $\left(\lambda_{1}(x, y), \lambda_{2}(x, y), \ldots, \lambda_{n}(x, y)\right) \in \Delta_{n-1}$ and $(x, y)=\sum_{i=1}^{n} \lambda_{i}(x, y)\left(a_{i}, b_{i}\right)$.

Let's define $f: K \rightarrow 2^{Y}$ by

$f\left(a_{i}, b_{i}\right)=c_{i}(i=1, \ldots, n)$ and

$f\left(\sum_{i=1}^{n} \lambda_{i}\left(a_{i}, b_{i}\right)\right)=\sum_{i=1}^{n} \lambda_{i} c_{i} \in T(x, y)$.

We show that $f$ is continuous.

Let $\left(x_{m}, y_{m}\right)_{m \in N}$ be a sequence which converges to $x_{0} \in K$, where $\left(x_{m}, y_{m}\right)=$ $\sum_{i=1}^{n} \lambda_{i}\left(x_{m}, y_{m}\right)\left(a_{i}, b_{i}\right)$ implies $a_{1}=a_{2}=\ldots=a_{n}=a$ or $b_{1}=b_{2}=\ldots=b_{n}=b$ and $\left(x_{0}, y_{0}\right)=\sum_{i=1}^{n} \lambda_{i}\left(x_{0}\right)\left(a_{i}, b_{i}\right)$ with $a_{1}=a_{2}=\ldots=a_{n}=a$ or $b_{1}=b_{2}=$ $\ldots=b_{n}=b$. By the continuity of $\lambda_{i}$, it follows that for each $i=1,2, \ldots, n$, $\lambda_{i}\left(x_{m}, y_{m}\right) \rightarrow \lambda_{i}\left(x_{0} . y_{0}\right)$ as $m \rightarrow \infty$. Hence $f\left(x_{m}, y_{m}\right) \rightarrow f\left(x_{0}, y_{0}\right)$ as $m \rightarrow \infty$, i.e. $f$ is continuous. 
Since $s: Y \rightarrow K$ is continuous, we obtain that $s \circ f: K \rightarrow K$ is continuous. According to Brouwer's fixed point theorem, there exists a point $x^{*} \in K$ such that $x^{*}=s \circ f\left(x^{*}\right)$ and then, $x^{*} \in s \circ T\left(x^{*}\right)$.

Theorem 13. (selection theorem). Let $Y$ be a non-empty subset of a topological vector space $F$ and $K \subset E_{1} \times E_{2}$, where $E_{1}, E_{2}$ are topological vector spaces. Suppose that $K$ is the biconvex hull of $\left\{\left(a_{1}, b_{1}\right),\left(a_{2}, b_{2}\right), \ldots,\left(a_{n}, b_{n}\right)\right\} \subset E_{1} \times E_{2}$. Let $T: K \rightarrow 2^{Y}$ be a weakly biconvex correspondence. Then, $T$ has a continuous selection on $K$.

In order to prove the existence of equilibrium, we need the following theorem:

Theorem 14. ([10]). Let $I$ and $J$ be any (possibly uncountable) index sets. For each $i \in I$ and $j \in J$, let $X_{i}$ and $Y_{j}$ be non-empty compact convex subsets of Hausdorff locally convex spaces $E_{i}$ and respectivelly $F_{j}$.

Let $X:=\prod X_{i}, Y:=\prod_{i \in I} Y_{j}$ and $Z:=X \times Y$.

For each $i \in I$ let $S_{i}: Z \rightarrow 2^{X_{i}}$ be a correspondence such that the set $W_{i}=\left\{(x, y) \in Z \mid S_{i}(x, y) \neq \emptyset\right\}$ is open and $S_{i}$ has a continuous selection $f_{i}$ on $W_{i}$.

For each $j \in J$ let $T_{j}: Z \rightarrow 2^{Y_{j}}$ be an upper semicontinuous correspondence with non-empty closed convex values.

Then there exists a point $\left(x^{*}, y^{*}\right) \in Z$ such that for each $i \in I$, either $S_{i}\left(x^{*}, y^{*}\right)=\emptyset$ or $x_{i}^{*} \in S_{i}\left(x^{*}, y^{*}\right)$, and for each $j \in J, y_{j}^{*} \in T_{j}\left(x^{*}, y^{*}\right)$.

As a consequence, we have the following:

Corollary 15. Let $I$ and $J$ be any (possibly uncountable) index sets. For each $i \in I$ and $j \in J$, let $X_{i}$ and $Y_{j}$ be non-empty compact convex subsets of Hausdorff locally convex spaces $E_{i}$ and respectivelly $F_{j}$.

Let $X:=\prod X_{i}, Y:=\prod_{i \in I} Y_{j}$ and $Z:=X \times Y$.

For each $i \in I$ let $S_{i}: Z \rightarrow 2^{X_{i}}$ be a correspondence such that the set $W_{i}=\left\{(x, y) \in Z \mid S_{i}(x, y) \neq \emptyset\right\}$ is the interior of the biconvex hull of $\left\{\left(a_{1}, b_{1}\right),\left(a_{2}, b_{2}\right), \ldots\right.$, $\left.\left(a_{n}, b_{n}\right)\right\} \subset Z$ and $S_{i}$ is weakly biconvex on $W_{i}$.

For each $j \in J$ let $T_{j}: Z \rightarrow 2^{Y_{j}}$ be an upper semicontinuous correspondence with non-empty closed convex values.

Then there exists a point $\left(x^{*}, y^{*}\right) \in Z$ such that for each $i \in I$, either $S_{i}\left(x^{*}, y^{*}\right)=\emptyset$ or $x_{i}^{*} \in S_{i}\left(x^{*}, y^{*}\right)$, and for each $j \in J, y_{j}^{*} \in T_{j}\left(x^{*}, y^{*}\right)$. 


\subsection{Kim's model of the generalized quasi-game and equilibrium theorems}

In this section, we study the following model of a generalized quasi-game.

Let $I$ be a nonempty set (the set of agents). For each $i \in I$, let $X_{i}$ be a non-empty topological vector space representing the set of actions and let's define $X:=\prod_{i \in I} X_{i}$; let $A_{i}, B_{i}: X \times X \rightarrow 2^{X_{i}}$ be the constraint correspondences and $P_{i}: X \times X \rightarrow 2^{X_{i}}$ the preference correspondence.

Definition 11. [10]. A generalized quasi-game $\Gamma=\left(X_{i}, A_{i}, B_{i}, P_{i}\right)_{i \in I}$ is defined as a family of ordered quadruples $\left(X_{i}, A_{i}, B_{i}, P_{i}\right)$.

In particular, when $I=\{1,2 \ldots n\}, \Gamma$ is called the n-person quasi-game.

Definition 12. [10]. An equilibrium for $\Gamma$ is defined as a point $\left(x^{*}, y^{*}\right) \in X \times$ $X$ such that, for each $i \in I, y_{i}^{*} \in c l B_{i}\left(x^{*}, y^{*}\right)$ and $A_{i}\left(x^{*}, y^{*}\right) \cap P_{i}\left(x^{*}, y^{*}\right)=\emptyset$.

If $A_{i}(x, y)=B_{i}(x, y)$ for each $(x, y) \in X \times X$ and $i \in I$, this model coincides with the one introduced by W. K. Kim [8].

If, in addition, for each $i \in I, A_{i}, P_{i}$ are constant with respect to the first argument, this model coincides with the classical one of the abstract economy and the definition of equilibrium is that given in [18].

Now, we state the following equilibrium theorem for generalized quasigames with correspondences which does not have continuity properties.

Theorem 16. Let $\Gamma=\left(X_{i}, A_{i}, B_{i}, P_{i}\right)_{i \in I}$ be a generalized quasi-game where $I$ is a (possibly uncountable) set of agents such that for each $i \in I$ :

(1) $X_{i}$ is a non-empty compact convex set in a Hausdorff locally convex space $E_{i}$ and denote $X:=\prod_{i \in I} X_{i}$ and $Z:=X \times X$;

(2) The correspondence $B_{i}: Z \rightarrow 2^{X_{i}}$ is non-empty, convex valued such that for each $(x, y) \in Z, A_{i}(x, y) \subset B_{i}(x, y)$ and $\mathrm{cl} B_{i}$ is upper semicontinuous;

(3) $\left(A_{i}, A_{i} \cap P_{i}\right)$ is a weakly biconvex pair of correspondences on $W_{i}$;

(4) the set $W_{i}:=\left\{(x, y) \in Z /\left(A_{i} \cap P_{i}\right)(x, y) \neq \emptyset\right\}$ is the interior of the biconvex hull of $\left\{\left(a_{1}, b_{1}\right),\left(a_{2}, b_{2}\right), \ldots,\left(a_{n}, b_{n}\right)\right\} \subset Z$;

(5) for each $(x, y) \in W_{i}, x_{i} \notin \operatorname{co} P_{i}(x, y)$.

Then there exists an equilibrium point $\left(x^{*}, y^{*}\right) \in Z$ for $\Gamma$, i.e., for each $i \in I, y_{i}^{*} \in \operatorname{cl} B_{i}\left(x^{*}, y^{*}\right)$ and $A_{i}\left(x^{*}, y^{*}\right) \cap P_{i}\left(x^{*}, y^{*}\right)=\emptyset$.

Proof. For each $i \in I$, we define $\Phi_{i}: Z \rightarrow 2^{X_{i}}$ by 


$$
\Phi_{i}(x, y)= \begin{cases}c o\left(A_{i} \cap P_{i}\right)(x, y), & \text { if }(x, y) \in W_{i} \\ \emptyset, & \text { if }(x, y) \notin W_{i}\end{cases}
$$

The restriction $A_{i} \cap P_{i / W_{i}}: W_{i} \rightarrow 2^{X_{i}}$ is a weakly biconvex correspondence. Then, applying Theorem 9 , we can obtain that there exists a continuous selection $f_{i}: W_{i} \rightarrow X_{i}$ such that $f_{i}(x, y) \in\left(A_{i} \cap P_{i}\right)(x, y)$ for each $(x, y) \in W_{i}$.

For each $j \in I$, we define $\Psi_{j}: Z \rightarrow 2^{X_{i}}$, by $\Psi_{j}(x, y)=\operatorname{cl} B_{j}(x, y)$ for each $(x, y) \in Z$.

Then $\Psi_{j}$ is an upper semicontinuous correspondence and $\Psi_{j}(x, y)$ is a nonempty, convex, closed subset of $X_{j}$ for each $(x, y) \in Z$.

According to Theorem 10, it follows that there exists $\left(x^{*}, y^{*}\right) \in Z$ such that for each $i \in I$, either $\Phi_{i}\left(x^{*}, y^{*}\right)=\emptyset$ or $x_{i}^{*} \in \Phi_{i}\left(x^{*}, y^{*}\right)$ and for each $j \in J, y_{j}^{*} \in \Psi_{j}\left(x^{*}, y^{*}\right)$.

If $x_{i}^{*} \in \Phi_{i}\left(x^{*}, y^{*}\right)$ for some $i \in I$, then $x_{i}^{*} \in \Phi_{i}\left(x^{*}, y^{*}\right)=\operatorname{co}\left(A_{i} \cap\right.$ $\left.P_{i}\right)\left(x^{*}, y^{*}\right) \subset \operatorname{co} P_{i}\left(x^{*}, y^{*}\right)$ which contradicts the assumption (5).

Therefore, for each $i \in I, \Phi_{i}(x, y)=\emptyset$ and then $\left(x^{*}, y^{*}\right) \notin W_{i}$. Hence, $\left(A_{i} \cap P_{i}\right)\left(x^{*}, y^{*}\right)=\emptyset$ and for each $i \in I, y^{*} \in \Psi_{i}\left(x^{*}, y^{*}\right)=\operatorname{cl} B_{i}\left(x^{*}, y^{*}\right)$.

Acknowledgment: This work was supported by the strategic grant POSDRU/89/1.5/S/58852, Project "Postdoctoral programme for training scientific researchers" cofinanced by the European Social Found within the Sectorial Operational Program Human Resources Development 2007-2013.

\section{References}

[1] R. Aumann, S. Hart, Bi-convexity and bi-martingales, Isr J Math 54, 2 (1986), 159-180.

[2] A. Borglin and H. Keiding, Existence of equilibrium action and of equilibrium:A note on the 'new' existence theorem. J. Math. Econom. 3 (1976), 313-316.

[3] X. Ding and He Yiran, Best Approximation Theorem for Set-valued Mappings without Convex Values and Continuity. Appl Math. and Mech. English Edition, 19, 9 (1998), 831-836.

[4] K. Fan, Fixed-point and minimax theorems in locally convex topological linear spaces. Proc. Nat. Acad. Sci. U.S.A. 38 (1952), 121-126.

[5] J. Gorski, F. Pfeuffer, K. Klamroth, Biconvex sets and optimization with biconvex functions: a survey and extension, Math. Meth. Oper. Res. 66 (2007), 373-407.

[6] C. J. Himmelberg, Fixed points of compact multifunctions. J. Math. Anal. Appl. 38 (1972), 205-207. 
[7] C. D. Horvath, Extension and selection theorems in topological vector spaces with a generalized convexity structure. Ann. Fac. Sci., Toulouse 2, (1993), 253-269.

[8] W. K. Kim, On a quasi fixed-point theorem, Bull.Korean Math. Soc. 40 (2003); No. 2, pp. 301-307.

[9] I. Lefebvre, A quasi fixed-point theorem for a product of u.s.c. or l.s.c. correspondences with an economic application, Set-Valued Anal. 9 (2001), 273-288.

[10] M. Patriche, Equilibrium for generalized quasi-games, Mathematical Reports, 10(60), 2 (2008), 185-195.

[11] M. Patriche, Equilibrium in abstract economies with weakly convex graph correspondences, Mathematical Reports, 10(60), 4 (2008), 359-373.

[12] W. Shafer and H. Sonnenschein, Equilibrium in abstract economies without ordered preferences. Journal of Mathematical Economics 2 (1975), 345-348.

[13] G. Tian, Fixed point theorems for mappings with noncompact and nonconvex domains, J. Math. Anal. Appl. 158 (1991) 161-167.

[14] X. Zheng, Approximate selection theorems and their applications. J. Math. An. Appl. 212 (1997), 88-97.

[15] K. Wlodarczyk, D. Klim, Fixed point and coincidence theorems for setvalued maps with nonconvex or noncompact domains in topological vector spaces, Abstr. Appl. Anal. (1) (2003) 1-18.

[16] K. Wlodarczyk, D. Klim, Equilibria and fixed points of sets-valued maps with nonconvex and noncompact domains and ranges, Nonlinear Analysis 65 (2006), 918-932.

[17] X. Wu, A new fixed point theorem and its applications. Proc. Amer. Math. Soc. 125 (1997), 1779-1783.

[18] N. C. Yannelis and N. D. Prabhakar, Existence of maximal elements and equilibrium in linear topological spaces. J. Math. Econom. 12 (1983), 233245.

[19] X. Z. Yuan, The Study of Minimax Inequalities and Applications to Economies and Variational Inequalities. Memoirs of the American Society, 625 (1988). 
Monica PATRICHE,

Department of Mathematics,

University of Bucharest,

14 Academiei Street, 010014 Bucharest, Romania.

Email: monica.patriche@yahoo.com 